\title{
Response to the Letter to the Editor
}

\section{Predictive Validity of FCE?}

Received 1 December 2008

In response to the concerns about our publication, the primary author of this study would like to address the following points:

Time lapse between data collection and reporting. While it is true that there was a significant time lapse between data collection and data reporting, we do not believe that significant changes occurred in the workers' compensation system that would affect the results of this research. Economic and social factors vary to some extent from patient to patient and employer to employer, regardless of the time frame between data collection and publication. These changes limit the ability to generalize the results of any research, including the citations listed in the editorial.

Reporting numbers of subjects rather than percentages. Both numbers and percentages were reported. Admittedly the number of subjects was small. This fact was pointed out as a limitation of the study by the authors. However, the number of subjects does not diminish the importance of the results of the study. In several of the studies cited in the editorial, actual follow up numbers are less than half of the study sample (a fact that could create significant bias) and the total number of those who were reached for follow up were not significantly higher than the number of subjects in our study.

Patients and therapists aware of recommendations. In the US workers' compensation system, it would be impossible to conduct testing without revealing the results of the test. Certainly the study patients knew the results and attempted to follow them, which is the purpose of an FCE. Our study examines whether or not they were able to follow the recommendations. As you correctly point out, many factors other than physical ones affect return to work. We found that the availability of temporary modified duty strongly affected return to work in this patient population.

No randomized controlled trial (RCT). An RCT is unrealistic in the US where FCE is considered a stan- dard of care with regard to return to work and disability determination. There are no insurance carriers that would permit such a study. I would like to point out that the references cited by Dr. Goss as important ones are also not RCTs. So it is surprising that this criticism is levied at our manuscript when those studies he cites are not RCTs.

Important literature not cited. The omission of the references cited by Dr. Gross was simply an oversight. The studies cited by Dr. Gross, where the FCE was found not to be a particularly strong predictor of successful return to work, involved the Isernhagen FCE. Research with one system cannot be generalized to all FCE systems. FCE systems vary significantly, particularly with regard to the scoring process. The approach used in the current study is the ErgoScience FCE, the Physical Work Performance Evaluation.

Interestingly, in one of the studies cited, FCE Performance Does Not Predict Sustained Return to Work in Claimants with Chronic Back Pain (2), only 6 patients met all the physical demands of the job, yet 37 of those followed up were working without worksite modifications. This would suggest that employers were ignoring the fact that these workers needed accommodation and were requiring them to perform physical demands for which they were not capable. In our view, this is an improper use of the FCE results and as such it is not surprising that there was little correlation between sustained return to work and FCE results. In another study cited (1), those who passed all FCE test items, had no recurrence of injury. This is a finding suggests that FCEs, when used properly, have a strong predictive ability of work sustainability. It is surprising that this finding was downplayed by the authors when they reported the results of this study.

FCE results do not predict work ability. We agree that physical abilities are not the sole predictor of return to work. However, we also believe that phys- 
ical functional abilities are an important component that significantly influences whether an employee can return to her/his job and that FCEs are a strong predictor of this relationship. We also believe that this study provides important preliminary evidence of the positive impact of an objective, reliable and valid FCE. Without using physical functional testing, the return-to-work decision becomes merely guesswork on the part of a physician who does not know the patient's functional abilities or the demands of the job. If work ability does not match job demands, then safe, sustainable return to work without injury is highly unlikely, regardless of the psychosocial fac- tors. In addition, we question the methodology and conclusions drawn in the studies cited as evidence that FCEs have little predictive ability for sustained work. When test results are improperly used, one cannot rightly claim that the test doesn't accomplish its intended purpose.
Deborah E. Lechner ErgoScience, Inc. Birmingham, AL, USA 